\title{
METHODS OF QUALITY CONTROL OF PHONOGRAMS DURING RESTORATION AND RECOVERY
}

\author{
Alex Grebin ${ }^{1}$, Ninel Levenets ${ }^{2}$, Volodymyr Shvaichenko ${ }^{3}$ \\ ${ }^{1}$ Department of Acoustic and Multimedia Electronic Systems, National Technical University of Ukraine «Igor Sikorsky Kyiv Polytechnic \\ Institute», Kyiv, Ukraine \\ ${ }^{2}$ Department of Acoustic and Multimedia Electronic Systems, National Technical University of Ukraine «Igor Sikorsky Kyiv Polytechnic \\ Institute», Kyiv, Ukraine

The object of research. Process of phonograms restoration and recovery are described. Investigated problem. Differences between some methods of quality control of phonograms during and after their restoration and recovery were investigated.

The main scientific results. An instrumental method for objective assessment of the quality of phonograms is proposed, based on a non-intrusive model with parametric modeling of the phonogram signal to assess the effect of an artifact on a phonogram.

The area of practical use of the research results. The results of the operational control of objective quality indicators of a real sound signal using virtual measuring instruments built into the software for working with sound are considered.

An innovative technological product: a technology for assessing the quality of phonograms in the process of restoration and recovery (R\&R), which makes it possible to objectively assess the quality of phonograms, taking into account artifacts of phonograms caused by the method of recording phonograms, the conditions of their storage, etc. enough high quality restored audio content.

Scope of application of the innovative technological product: studio of restoration and recovery of sound phonograms on analog media, recording studios, technological processes of conversion and processing of sound programs, archives of radio and television recordings.

(C) The Author(s) 2021. This is an open access article under the CC BY license http://creativecommons.org/licenses/by/4.0).

\section{Introduction}

\section{1. The object of research}

The restoration and recovery of phonograms is an important stage in the preservation of the sound heritage of mankind. As a rule, most of the phonograms during the storage period lose their quality indicators, which must be restored during the restoration process.

To control the quality of phonograms during the restoration process, subjective and objective methods are used, which can be carried out both in operational and non-operational modes.

\section{2. Problem description}

Subjective methods involve auditory control of the sound of phonograms using high-quality loudspeakers in a specialized, acoustically designed room - a control studio and, most importantly, are carried out with the assistance of experts.

Such methods require sufficient funding and a lot of time to implement them, although it is impossible without them.

Together with subjective methods, objective control of the quality indicators of the phonogram is carried out.

To carry out objective quality control in real conditions during the restoration and restoration of phonograms, hardware and software tools for measuring the parameters of phonograms 
are used, and instrumental methods called quality models have been developed. Such methods require the presence of specialized software designed to automatically determine the quality of the signal reproduced from the phonogram.

A significant drawback of the overwhelming majority of modern studies aimed at objectifying the assessment of the quality of distorted signals is the predominant interest in "intrusive" indicators, while the tasks of restoring phonograms require the use of either "non-intrusive" or so-called "parametric" indicators.

\section{3. Suggested solution to the problem}

To solve the problem of performing an objective assessment of the quality of phonograms during and after R\&R Ph-process, the paper proposes an improvement in the method for assessing the quality of phonograms, based on a non-intrusive model with recognition not of the information component of phonograms, as in most cases of an objective assessment of sound quality, but of phonogram artifacts and determining the effect of artifacts on informational component of a phonogram.

\section{Materials and methods}

The quality of phonograms when performing the process of restoration and recovery of phonograms $(\mathrm{R} \& \mathrm{R} \mathrm{Ph})$ is, first of all, assessed by the presence, quantity and significance of artifacts in the phonogram, and, secondly, by the quality and properties of the useful informational component of the phonogram.

\section{1. Features of the subjective method for evaluation the quality of phonograms before} and after the $\mathbf{R} \& \mathbf{R} \mathbf{P h}$-process

Taking into account the technology of the $\mathrm{R} \& \mathrm{R} \mathrm{Ph}$ conducting work with phonograms, when at the initial stage of restoration artifacts are assessed and removed, and then the quality indicators of information are improved, the model for an objective assessment of the quality of phonograms should be based on the definition of artifacts, and then the quality of the useful component.

The peculiarity of the quality control of phonograms when performing the $\mathrm{R} \& \mathrm{R} \mathrm{Ph}$ is that, firstly, the sound engineer-restorer does not know the original quality indicators of sound and relies entirely on its own experience in evaluation the quality, and secondly, as a rule, there is no original sound of the sound fragment and does not with what to compare the quality; thirdly, the final result of the sound quality depends on the presence and "magnitude" of phonogram artifacts, as well as on the further use of the phonogram.

Considering also that the R\&R Ph-process is on the verge of creativity and technology, the dictum on the basis of which the perceptual model was compiled is not applicable to the $\mathrm{R} \& \mathrm{R}$ Ph-process - "subjective auditory tests take a long time, are expensive and unsuitable for everyday use, and therefore objective, computer-based methods of quality assessment are needed sound" [1].

In this regard, subjective control of the quality of phonograms is mandatory at the first stages by the sound engineer-restorer itself, and at the final stage - by experts.

Subjective control involves an assessment of the sound quality by listening to a phonogram through high-quality loudspeakers in an acoustically decorated room and using at least 5 experts.

During the R\&R Ph-process, the processing of the phonogram when removing phonogram artifacts is carried out in several stages, including: removing clicks, noise, level unevenness, and frequency response correction. In this case, the time spent on the execution of each operation throughout the entire fragment can take from hours to several working days. The main subjective control of the quality of phonograms is performed by the sound engineer-restorer.

Among the criteria that must be taken into account in the process of evaluation the sound quality of phonograms, in accordance with the OIRT protocol, the following are defined as the main ones [2]:

1) Spatial impression;

2) Transparency; 
3) Musical balance;

4) Coloring sound, timbre;

5) Interference;

6) Artistic form;

7) Arrangement;

8) Technique of sound;

9) Stereo impression;

10) Main impression.

To carry out the R\&R Ph-process some of these criteria must be supplemented by the presence of phonogram artifacts in the "interference" clause with interference interpretation - structure noise, clicks, modulation noise, roar noise, copy effect, detonation. Instead of "sound technique" use "restoration technique", instead of "arrangement" apply signal processing according to the criterion of sound improvement.

The objective parameters by which the quality of the phonogram is assessed are the signal level, frequency response, nonlinear distortions, signal-to-noise ratio, dynamic range, phase and level relationships between channels of a stereo signal, penetration from a channel into a channel of a stereo signal.

\section{2. Instrumental methods for evaluation the quality of phonograms}

The instrumental method for evaluation the quality of phonograms is based on a mathematical model that establishes the relationship between the perception of sound and a physical quantity, an example of which can be the Weber-Fechner law on the logarithmic law of perception of sound signals by a person.

Instrumental methods are generally classified into three groups [3]:

- parameter-based models, which provides a preliminary description of the parameters by which the quality assessment is made. Such models predict the perceived signal quality;

- models based on signals, which provides for the formation of test signals, the quality of which is evaluated after passing through the sound transmission path and compared with the input. These models assume comparison of signals after passing through the sound transmission paths and the use of certain signal processing devices;

- packet-based models involve evaluating certain signal processing conditions in a set of parameters or evaluating the effect of transmission channels on the resulting signal, and such models allow analyzing signal quality by several parameters simultaneously.

Parameter-based models allow evaluating the value of each signal parameter independently of each other, and then, combining them into a general picture, determine the quality of the phonogram. For such a model, it is necessary to specify a list of parameters that will determine the quality of the phonogram. From the parameter list, the parameter-based models are able to predict the quality of the $R \& F$ results.

The packet-based model is designed to assess the quality of the signal in terms of several parameters, including equipment, media, information component of a phonogram. The packet-based models allow the evaluation of the signal artifacts themselves, and the need for a reliable instrumental model.

Among the instrumental models for evaluation the quality based on the parameters of the signal of phonograms during their restoration, it is possible to offer the E-model.

The E-model (electronic model) provides for a certain rating of the signal, as a parameter that may deteriorate during the conversion process.

In accordance with the E-model, the signal to be restored at the initial stage even during recording had a rating of $R_{0}[3]$. After recording on the appropriate media, after repeated playback and long-term storage, the signal rating changes significantly and can be written as follows

$$
R=R_{0}-I_{S}-I_{d}-I_{e}+A
$$

where $I_{S}$ are artifacts determined by the recording location, acoustic conditions of recording, noises that appear at the recording location, etc., $I_{d}$ are artifacts determined by equipment short- 
comings, $I_{e}$ are artifacts determined by the storage conditions of the phonogram, $A$ is an advantage parameter showing the advantages one phonogram in relation to another.

The parameter-based model does not always allow to evaluate the signal itself with useful information at the output of the system while directly monitoring the quality of the signal itself. In addition, parameter-based models are less reliable in the presence of multidisciplinary and combined artifacts. In this case, it is better to use instrumental methods based on the signal [3].

These models are divided into two groups:

a) intrusive models;

b) non-intrusive models. Intrusive ones are based on a double measurement method, which uses a reference clean signal $x(t)$ and, accordingly, a signal $y(t)$ degraded by artifacts. The intrusive method has the ability to compare a reference signal with a signal that has passed through a transmission channel or a recording channel.

Non-intrusive models are one-sided and assume work only with resulting signals, that is, signals at the output of the system, namely, degraded signals. Non-intrusive models do not have the ability to compare the output signal with a reference, since such does not exist.

The structural model of the phonogram recording and restoration process using intrusive and non-intrusive models is shown in Fig. 1 [3].

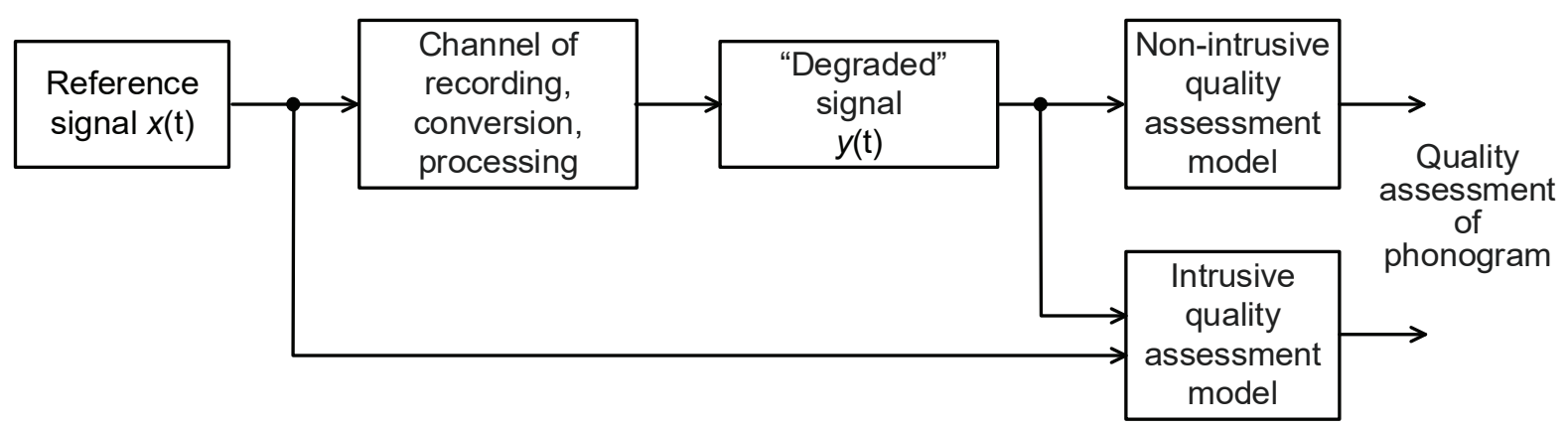

a

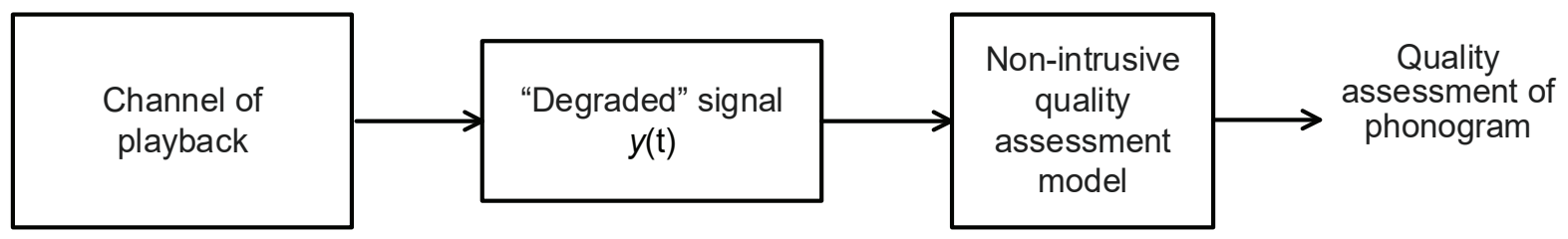

b

Fig. 1. Structural model for evaluation the quality of phonogram creation in the presence of a reference signal: $a$-during recording of phonograms in the presence of only a playback signal; $b$-during restoration of phonograms in the presence of only a playback signal

When an obvious artifact (distortion, extraneous noise, etc.) appears with the intrusive method, it is possible to compare it with a reference signal and use processing tools to reduce artifacts. The presence of an artifact with a non-intrusive method does not always indicate the artifact itself, because in some cases the artifact itself is introduced by the performer as an effect.

Non-intrusive signal parameter control models rely on two different approaches - a priori and source-based. Both approaches are based on signal degradation and can describe either the perceptual characteristics of the signal or the physical characteristics.

The a priori approach provides that the signal is characterized by a set of known signal artifacts in several parameters, which are stored in the measuring system at the path output. The service non-intrusive measuring device In-service Non-intrusive Measurement Device (INMD) is used, which quantitatively determines the physical characteristics of the signal, such as signal level, 
noise, delay, etc. With this device, it is possible to predict the signal quality when perceived on the receiving side using, for example, the parametric E-model [3].

Another type of a priori non-intrusive models uses the probability that the damaged sound signal of the phonogram was reproduced at the initial level of its creation by natural sources such as voice, musical instruments, natural noise signals, which have certain features associated with the physiological rules of voice creation, sound production of musical instruments and others, and with which they could be compared. The derived parameters are then combined and displayed on the quality bar.

The sourcing approach assumes the presence of artificially generated reference signals that are selected from parameters that characterize the degraded signal. Then the artificially created standard is compared with the degraded signal.

This approach, like the a priori approach, is based on machine learning techniques. Moreover, in this case, the number of artificially created parameters is much larger, and the range of possible distortions is wider than in the a priori approach.

\section{The aim and objectives of research}

The proposed methods of objective control of sound programs such as: PEAQ method [4], method objective assessment of Perceptual Audio Quality Using ViSQOLAudio [5], non-intrusive methods for assessing sound quality [6-8] and others described in [3,5], are not always suitable for the R\&R Ph-process, since they consider purely speech signals or channels of speech transmission. In [9], a conceptual model of the $R \& R$ Ph- process is given taking into account the quality control of phonograms, but the control method is not displayed. More suitable for $\mathrm{R} \& \mathrm{R}$ Ph-process is the method of subjective assessment of sound quality [2] with the difference in the research conducted not by a group of experts, but by the director-restorer and changing some subjective criteria of sound quality.

Objective assessment of the quality of a sound program usually requires the use of intrusive quality assessment models.

The principle of objective assessment of sound quality using PEAQ technology consists in software modeling of characteristics inherent in human perception of a sound signal, followed by an assessment of differences in the sound of the output signal of the system or the sound path compared to the original (test) signal [4]. The structure of the PEAQ method is a sequential inclusion of a psychoacoustic model and a cognitive model. The input signals for the psychoacoustic link in the PEAQ structure are the reference (original) signal and the real degraded (distorted) features of the signal transmission channel. They are compared for different psychoacoustic traits and for each trait its own output variable (MOV) is created, which, in accordance with the cognitive analysis, forms the final quality assessment in points (ODG) from " 0 " (the deterioration is imperceptible) to " -4 " (the deterioration is very annoying) [4].

For PEAQ, the definition of the maximum noise level that a person does not perceive is used as the basic principle of constructing a perceptual model [4]. Moreover, this level can change depending on the time and frequency characteristics of the signal and, accordingly, the limit of recognition of these noises by the human hearing will change. The PEAQ method is based on evaluating the masking of a useful (masked) informative signal by a harmful noise (masking) signal that coincides with it in time, taking into account the spectral composition of the masked and masking and the relative position on the time scale [10].

The PEAQ method, like other methods of objective assessment of sound quality, is intended primarily to assess the quality of the transmission of sound programs at the output of sound transmission channels, at the output of signal conversion devices, in networks and sound distribution systems, and it is mandatory to compare the output signal with the reference test signal.

The purpose of the research is to improve the method of non-intrusive quality assessment in relation to the R\&R Ph-process.

Since an objective assessment of the quality of the reconstructed signal is necessary to assess the quality of sound in the R\&R Ph-process, namely, an assessment of the remnants of artifacts, their influence on the informative component of the audio signal and the results of improving the signal in comparison with the original distorted presence of artifacts. Those. an inverse assessment is assumed, the inverse approach is not an analysis of the presence of 
distortions created by the sound transmission channel, but an analysis of the absence of distortions after the R\&R Ph-process.

To achieve the aim of research, the following objectives were set and solved:

a) to improve the instrumental method of objectively evaluation the quality of phonograms, based on a non-intrusive model with parametric modeling of the phonogram signal to assess the effect of an artifact on a phonogram;

b) experimentally, using the example of a simulation model and virtual measuring instruments built into the software, to confirm the effectiveness of the improved method based on the results of operational control of the objective parameters of phonograms;

c) to generalize and develop the main criteria for the quality of phonograms for subjective and objective evaluation.

\section{Improvement of the method of objective evaluation of the quality of phonograms}

For the R\&R Ph-process the PEAQ technology is not always suitable, since the parameters and properties of the original useful signal are unknown, therefore, it is preferable to use the method for evaluation the sound quality of phonograms after the R\&R Ph-process, taking into account the presence of artifacts in the phonogram.

For this, it is desirable to model not the perceptual characteristics of hearing, as is done in PEAQ, but noises and other artifacts inherent in information carriers, and as a result of quality assessment to evaluate and compare the presence of an artifact, its level and changes in the useful signal.

In contrast to the perceptual evaluation of audio quality (PEAQ), for evaluation the quality of sound in the R\&R Ph-process, it is proposed to use the model of artifact evaluation of audio quality (AEAQ). For this model, the phonogram is reproduced by an appropriate device and the signal is fed simultaneously to the noise model and the psychoacoustic model (Fig. 2). The noise model compares possible phonogram artifacts with a phonogram bank compiled from possible artifacts of different recording media, taking into account spectral, level and temporal characteristics. The psychoacoustic model, as in the perceptual model, simulates the psychoacoustic features of a person's perception of sound with division into different features and determines the influence of the corresponding artifact on the useful signal. The result of the analysis can also be a display of the presence of a certain artifact, its individual parameters (level, spectrum, frequency in the phonogram), and a qualitative numerical assessment.

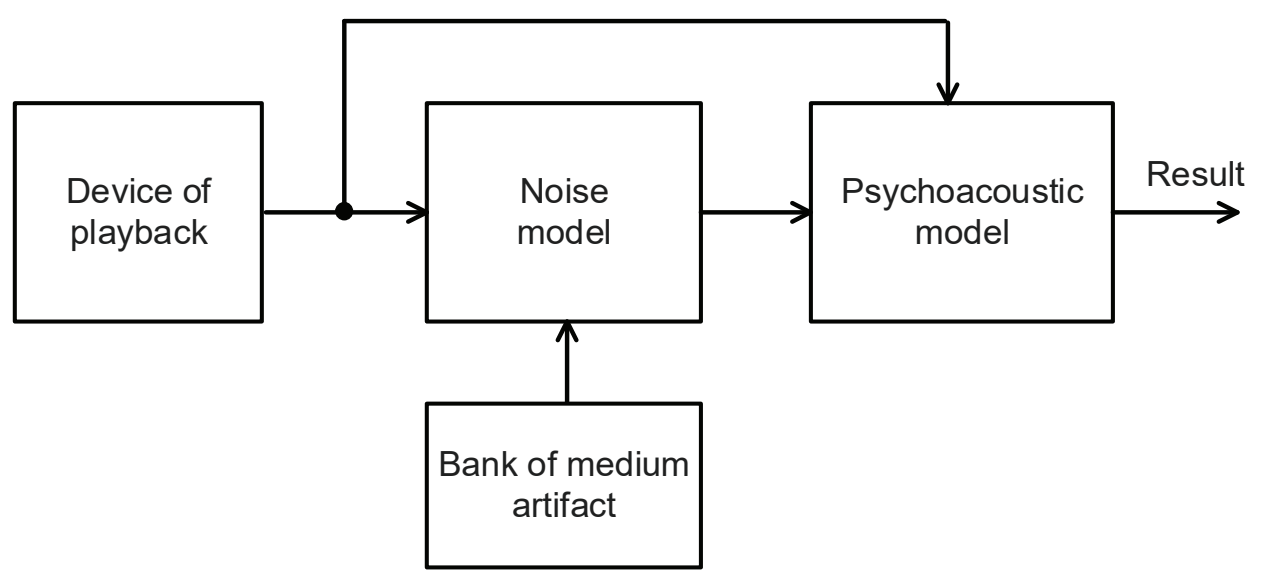

Fig. 2. Structure of the AEAQ model algorithm

The principle of the AEAQ model lies in the software simulation of various phonogram artifacts with the subsequent transformation of the output values of the artifact comparison with their influence on the useful signal. Based on the output resulting values, the assessment can be interpreted, as for PEAQ [4], on the scale: 0 - the effect of the artifact is not perceived; $(-1)$ - the effect of the artifact is insignificant (not annoying); $(-2)$ - existing artifacts are slightly annoying; (-3) - artifacts are unpleasant when listening; (-4) - artifacts are significant and very unpleasant to perceive. 
To implement the model of objective assessment of the signal quality in the R\&R Ph-process, it is possible to apply the method of parametric signal coding, which is used in compression systems for digital audio data of the type of MPEG-4 [11]. It is known that a real sound signal in a sinusoidal model can be written as

$$
x(t)=\sum_{i=1}^{I} A_{i}(t) e^{j \varphi_{i}(t)}
$$

where $A_{i}(t)$ is the amplitude of the $i$-th spectral component depending on time, $\varphi_{i}(t)$ is its phase, $\varphi_{i}(t)=\int_{-\infty}^{t} \omega_{i}(\tau) d \tau, i$ is the number of spectral components, $\omega_{i}(\tau)$ is instantaneous frequency of the spectral component versus time.

Taking into account the artifacts of the recording media, the phonogram signal subject to the $\& \mathrm{R}$ Ph-process can be written as

$$
x(t)=\sum_{i=1}^{I} A_{i}(t) e^{j \varphi_{i}(t)}+y(t)
$$

where $y(t)$ are the noise components of the signal, the so-called phonogram artifacts, which are determined by the recording medium $n(t)$, the storage conditions of the phonogram $k(t)$, and other factors (the conditions of the initial recording and the playback equipment) $m(t)$. Taking into account the artifacts of the phonograms, the phonogram signal can be recorded in the form

$$
x(t)=\sum_{i=1}^{I} A_{i}(t) e^{j \varphi_{i}(t)}+y(t)=\sum_{i=1}^{I} A_{i}(t) e^{j \varphi_{i}(t)}+n(t)+k(t)+m(t)+C,
$$

$C$ is a function that determines the presence of artifacts in the soundtrack. In discrete-time representation, taking into account the tonal components of the signal and noise components, the signal of a real phonogram can be written in the form

$$
x(n)=\sum_{i=1}^{I} A_{i}(n) \cos \left[\varphi_{i}(n)\right]+y(n),
$$

where $\sum_{i=1}^{I} A_{i}(n) \cos \left[\varphi_{i}(n)\right]$ is the sum of spectral components with time-varying values of am plitudes, frequencies, phases of the useful part of the signal, $y(n)$ are the noise components of the signal, including phonogram artifacts. With the R\&V of the useful signal of the phonogram, the signal is filtered by removing the artifact, as a result, there is a recovered signal

$$
x^{\prime}(t)=x(t)-y(t)
$$

This signal differs from the input signal, since it is not possible to completely restore the initial parameters.

When assessing the sound quality of phonograms, the analysis of individual parameters of the useful part of the phonogram and artifacts takes place, and as a result, the conditional gradations of artifacts and their influence on the useful signal are displayed. The principle of parametric modeling of the process of objective assessment of the quality of a phonogram is as follows. The input audio signal, damaged by phonogram artifacts, enters the input of the signal dividing unit into components, where the signal is divided into tonal and noise components (Fig. 3). After that, the tonal components are divided into harmonic and individual high-level [12]. Next, the values of the current frequencies, phases and amplitudes of each of their tonal components are estimated, and for the noise components, their energy levels in certain frequency bands are determined.

The values of the parameters of the tonal and noise components of the signal are compared with the psychoacoustic parameters and displayed by conventional measuring devices as the difference between the signal and the artifact that can be perceived by a person. 


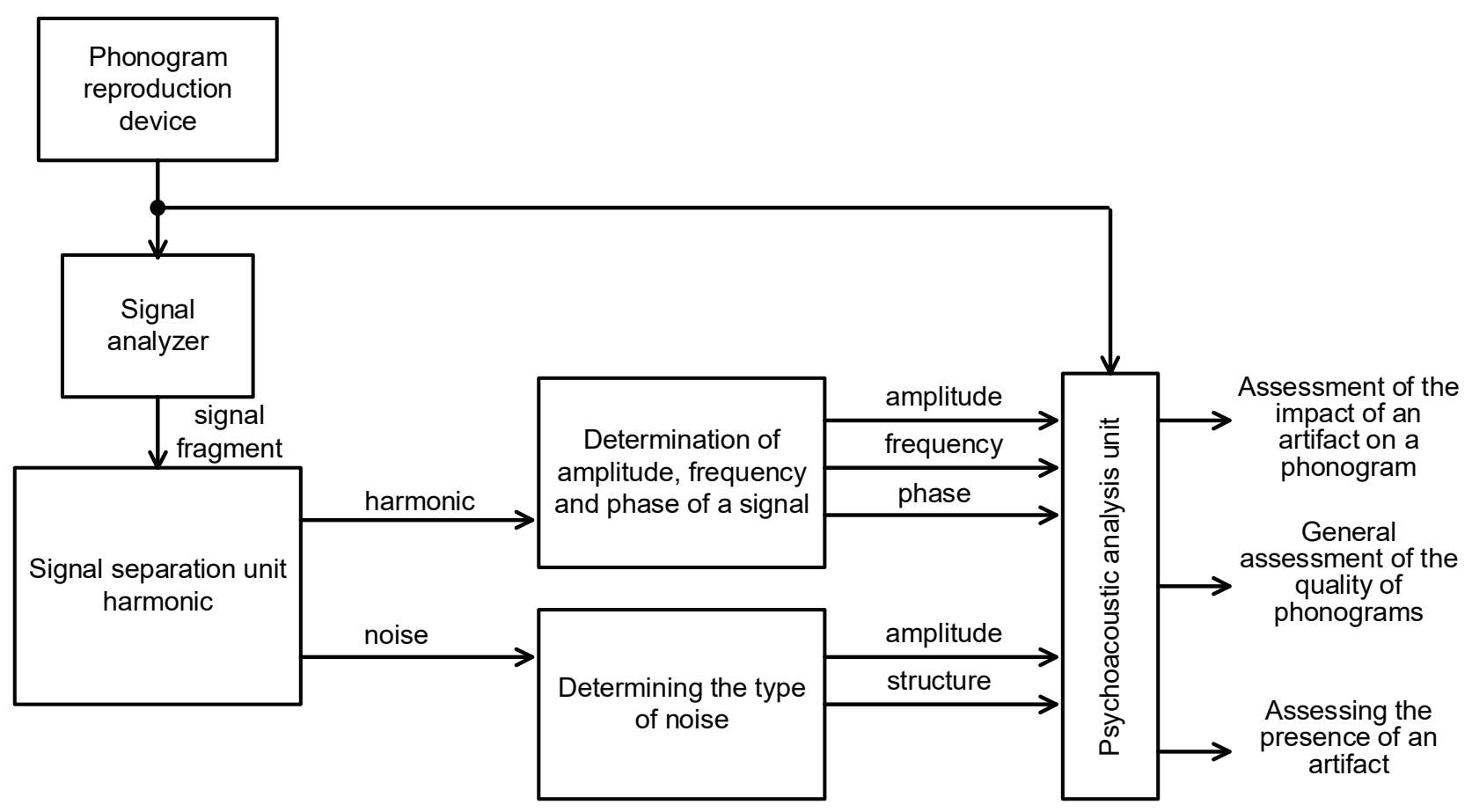

Fig. 3. Structure of parametric modeling of a phonogram signal to assess the effect of an artifact on a phonogram

\section{Results}

In reality, objective operational control includes monitoring:

1) waveform;

2) signal level using level indicators;

3) monitoring the frequency response of the signal using a spectrum analyzer;

4) phase relationships between the signals of the left and right channels of a stereo signal using a phase meter;

5) dynamic range, RMS signal level and other signal level parameters using statistical panels, etc.

By the waveform that is displayed in the main window of any program that works with sound, it is possible visually to determine the average level, signal peaks, the presence of clipping, pauses, useful signal, temporal parameters of phonogram fragments, signal dropouts, the presence of some phonogram artifacts [13].

Generally speaking, the main criteria for the quality of phonograms for an objective assessment when carrying out restoration and restoration work with phonograms that can be determined on-line are: signal and artifact level, signal and artifact frequency response, dynamic range, phase relationships between stereo channels.

Fig. 4. shows the main window of the Adobe Audition program with the display of operational tools for objective assessment of the signalogram quality.

The level meter allows to measure the average level and peak level of the signal, both useful and artifacts, in real time. The signal level at the peaks should not exceed $0 \mathrm{~dB}$, and the average level should not exceed the crest factor value for the corresponding sound programs (on average, $-12 \ldots-24 \mathrm{~dB}$ ). The level of noise and other artifacts of the phonogram should be as low as possible, not higher than $-60 \mathrm{~dB}$, which approximately corresponds to the threshold of audibility of the noise at the corresponding acoustic level of the phonogram reproduction. Using the level indicator, it is possible to calculate the signal-tonoise ratio as the difference between the signal and noise levels

$$
\mathrm{SNR}=N_{\text {sign }}-N_{\text {noise }}, \mathrm{dB},
$$

measuring the signal level at the point of the maximum level of the useful signal and in the pause, for example, between fragments of a phonogram. 


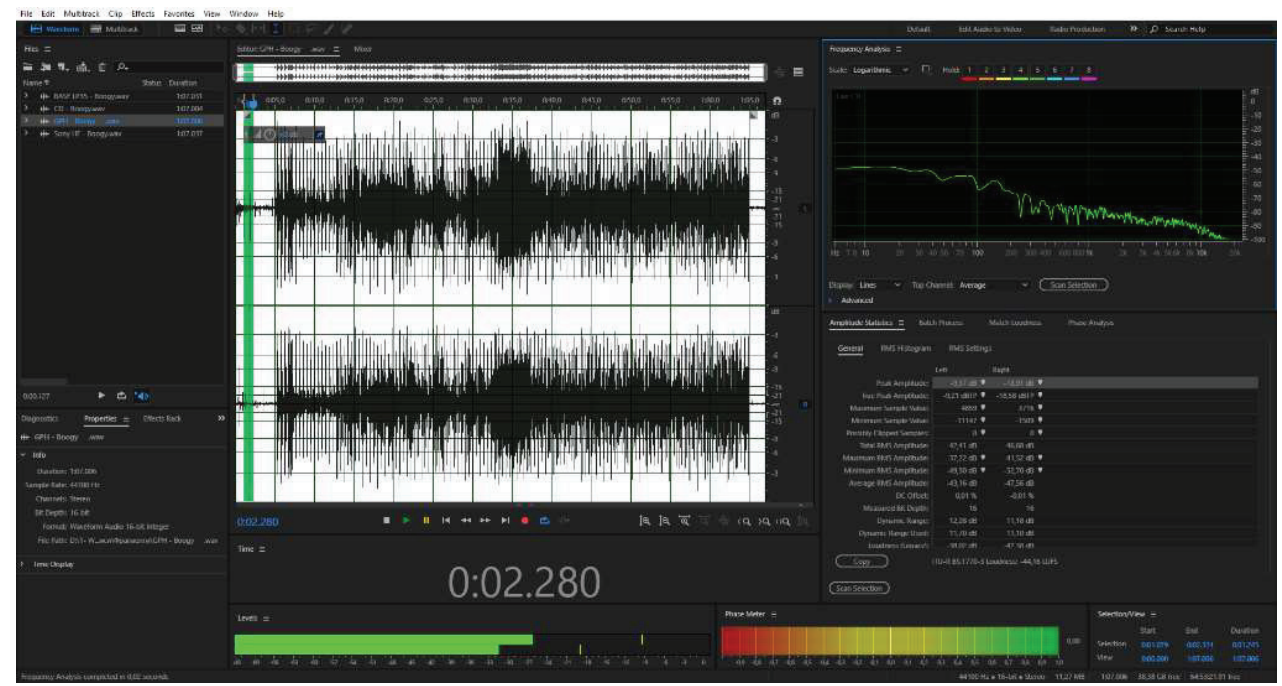

Fig. 4. Screenshot of the main window of the Adobe Audition program with the display of parameter values by built-in measuring tools

Fig. 5, $\boldsymbol{a}, \boldsymbol{b}$ shows the spectra of the information signal of several phonograms and phonogram artifacts. Fig. 6, $\boldsymbol{a}, \boldsymbol{b}$ show histograms of the information signal of the phonogram and phonogram artifacts.

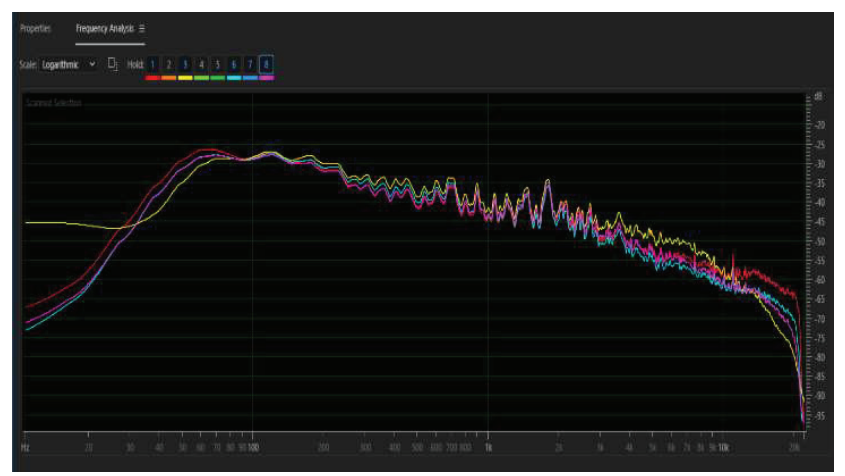

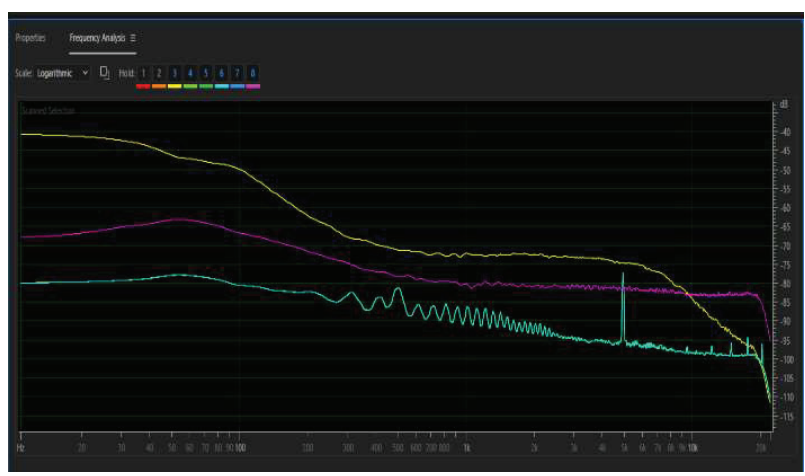

$b$

Fig. 5. Screenshot of the spectrum analyzer window of the Adobe Audition program with displaying the frequency response: $a$ - of the information signal of several phonograms; $b$ - of the artifacts of these phonograms

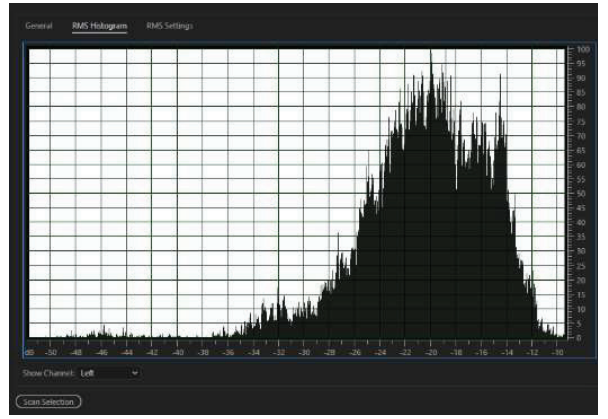

$a$

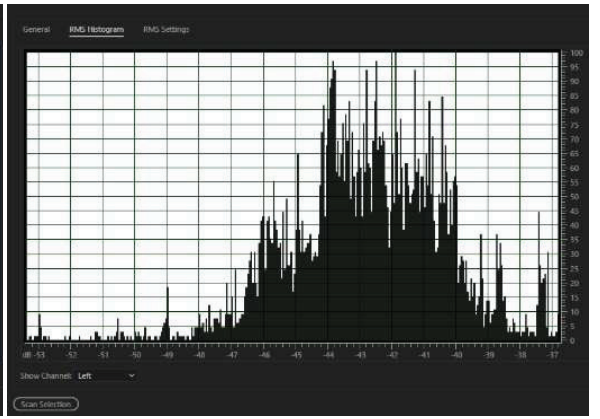

$b$

Fig. 6. Screenshot of the Amplitude Statistics window, RMS Histogram of the Adobe Audition program with displaying the histogram: $a$ - of the information signal of the phonogram; $b-$ of the artifacts of the phonograms 
Based on the results of the analysis of the frequency response of the information signal of the phonogram (Fig. 6, a) and phonogram artifacts (Fig. 7), it is possible to determine the signal/artifact ratio at different frequencies, which is important for operational control of phonograms in the $\mathrm{R} \& \mathrm{R} \mathrm{Ph}$-process. So, in accordance with the screenshot, the signal level at a frequency of $100 \mathrm{~Hz}$ is $-30 \mathrm{~dB}$, and the noise level is $-70 \mathrm{~dB}, \mathrm{SNR}=40 \mathrm{~dB}$, the signal level at a frequency of $10 \mathrm{kHz}$ is $-60 \mathrm{~dB}$, and the noise level is $-85 \mathrm{~dB}, \mathrm{SNR}=25 \mathrm{~dB}$. As it is possible to see, at a frequency of $100 \mathrm{~Hz}$, the SNR is greater than at a frequency of $10 \mathrm{kHz}$, which indicates the need to reduce noise at high frequencies to a greater extent than at low frequencies, although at frequencies below $50 \mathrm{~Hz}$, additional noise processing is also required downward to reduce their visibility.

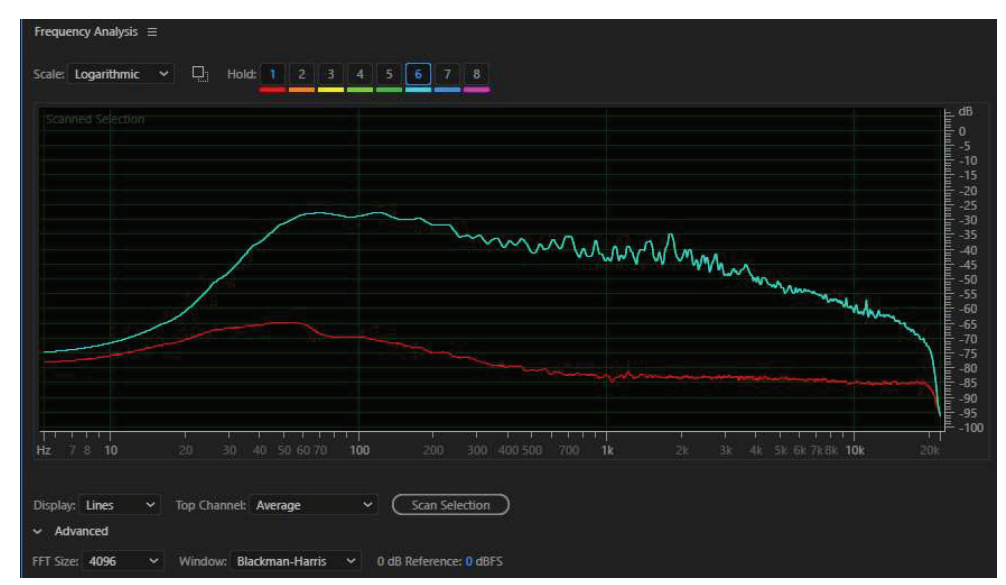

Fig. 7. Screenshot of the spectrum analyzer window of the Adobe Audition program with displaying the frequency response of the information signal of the phonogram (blue graph) and phonogram artifacts (red graph) findings

\section{Discussion}

The reliability of the measurement of operational parameters is shown in the screenshots. During restoration, knowledge of these parameters allows to determine the amount of work to remove certain artifacts (in this case, noise).

The disadvantages of the proposed improvement of the method include insufficient versatility in relation to possible artifacts and the need to create a bank of artifacts of phonograms of different carriers.

Most methods for assessing the quality of sound [3, 5] are, first of all, assessing the quality of audio information that passes through the communication channels that distort the signal. The proposed method, first of all, evaluates the artifacts of phonograms on which useful sound information is recorded. This is the main feature of methods for assessing the quality of sound information.

The need for specialized software can be called a limitation of the use of the proposed method for assessing the quality of phonograms.

Therefore, the main areas of further research are:

a) development of a software algorithm for the possible automation of the artifact identification process, and as a result of the information carrier itself;

b) study of the artifact value dependence on the possible types and parameters of processing during $\mathrm{R} \& \mathrm{R}$ Ph-process, on the quality of the information component of the phonogram.

\section{Conclusions}

1. The proposed model is based on the comparison of the artifacts of the investigated phonogram with the bank of artifacts, as well as the qualitative indicators of the investigated phonogram with psychoacoustic parameters, and displaying the assessment of the effect of artifacts on the useful information component of the phonogram.

2. On the basis of the methods of objective assessment of the quality of phonograms of some authors, as well as the PEAQ technology, described in the work, an improved method of opera- 
tional subjective control of parameters based on an expert assessment of parameters by a sound engineer-restorer is proposed.

3. Examples of operational control of objective parameters of a phonogram are considered, such as: signal and artifact level, signal and artifact frequency response, dynamic range, phase relationships between stereo channels using built-in software measuring tools.

4. The reliability of the measurement of operational parameters is shown in the screenshots. During restoration, knowledge of these parameters allows to determine the amount of work to remove certain artifacts (in this case, noise).

\section{References}

[1] Campbell, D., Jones, E., Glavin, M. (2009). Audio quality assessment techniques - A review, and recent developments. Signal Processing, 89 (8), 1489-1500. doi: http://doi.org/10.1016/j.sigpro.2009.02.015

[2] ITU-R Recommendation BS.1284-2 (2019). General methods for the subjective assessment of sound quality. International Telecommunications Union. Geneva, 18. Available at: https://www.itu.int/dms_pubrec/itu-r/rec/bs/R-REC-BS.1284-2201901-I!!PDF-E.pdf

[3] Cote N. (2011) Integral and Diagnostic Intrusive Prediction Speech Quality. T-Labs Series in Telecommunication Services. Berlin-Heidelberg: Springer-Verlag, 267. doi: http://doi.org/10.1007/978-3-642-18463-5

[4] ITU-R Recommendation BS.1387 (2001). Method for objective measurements of perceived audio quality. International Telecommunications Union. Geneva. Available at: https://www.itu.int/dms_pubrec/itu-r/rec/bs/R-REC-BS.1387-1-200111-I!!PDF-E.pdf

[5] Sloan, C., Harte, N., Kelly, D., Kokaram, A. C., Hines, A. (2017). Objective Assessment of Perceptual Audio Quality Using ViSQOLAudio. IEEE Transactions on Broadcasting, 63 (4), 693-705. doi: http://doi.org/10.1109/tbc.2017.2704421

[6] Avila, A. R., Gamper, H., Reddy, C., Cutler, R., Tashev, I., \& Gehrke, J. (2019). Non-intrusive Speech Quality Assessment Using Neural Networks. ICASSP 2019 - 2019 IEEE International Conference on Acoustics, Speech and Signal Processing (ICASSP). Brighton, 631-635. doi: http://doi.org/10.1109/icassp.2019.8683175

[7] Jia, X., Li, D. (2020). A Deep Learning-Based Time-Domain Approach for Non-Intrusive Speech Quality Assessment, 2020 Asia-Pacific Signal and Information Processing Association Annual Summit and Conference (APSIPA ASC). Auckland, 477-481.

[8] Shan, Y., Wang, J., Xie, X., Meng, L., Kuang, J. (2018). Non-intrusive Speech Quality Assessment Using Deep Belief Network and Backpropagation Neural Network. 2018 11th International Symposium on Chinese Spoken Language Processing (ISCSLP). doi: http://doi.org/10.1109/iscslp.2018.8706696

[9] Hrebin, O. P., Levenets, N. F., Shvaichenko, V. B. (2018). Model protsesu vidnovlennia informatsii z ushkodzhenykh nosiiv. Visnyk Kyivskoho natsionalnoho universytetu tekhnolohii ta dyzainu. Seriia: Mekhatronni systemy. Enerhoefektyvnist ta resursozberezhennia, 2 (120), 26-37.

[10] Tekhnolohyia PEAQ. Available at: https://audiophilesoft.ru/publ/theory/peaq/6-1-0-325

[11] ISO/IEC 14496-3 Information technology - Coding of audio-visual objects - Part 3: Audio (2005). Available at: https://www. iso.org/ru/standard/42739.html

[12] Kovalgin, Iu. A., Vologdin, E. I. (2013). Audiotekhnika. Moscow: Goriachaia liniia - Telekom, 742.

[13] Hrebin, O. P., Levenets, N. F. (2019). Zastosuvannia informatsiinykh tekhnolohii dlia kontroliu parametriv zvukovykh syhnaliv pry stvorenni audiovizualnoi produktsii. Informatsiini tekhnolohii v kulturi, mystetstvi, osviti, nautsi, ekonomitsi ta biznesi. Kyiv: Vydavnychyi tsentr KNUKiM, 2, 236-238. Available at: https://issuu.com/kn.knukim/ docs/ 2019 